\title{
Schlieren and dye laser resonance absorption photographic investigations of KrF excimer laser-ablated atoms and molecules from polyimide, polyethyleneterephthalate, and aluminum
}

\author{
Peter L. G. Ventzek, ${ }^{a}$ Ronald M. Gilgenbach, Chi Hong Ching, and Roger A. Lindley \\ Intense Energy Beam Interaction Laboratory, Nuclear Engineering Department, \\ The University of Michigan, Ann Arbor, Michigan 48109-2104
}

(Received 13 February 1992; accepted for publication 19 May 1992)

\begin{abstract}
Hydrodynamic phenomena from $\mathrm{KrF}$ excimer laser ablation $\left(10^{-3}-20 \mathrm{~J} / \mathrm{cm}^{2}\right)$ of polyimide, polyethyleneterephthalate, and aluminum are diagnosed by, schlieren photography, shadowgraphy, and dye laser resonance absorption photography (DLRAP). Experiments were performed both in vacuum and gaseous environments $\left(10^{-5}-760\right.$ Torr air, nitrogen, and argon). In vacuum, ablation plumes are observed to expand like a reflected rarefaction wave. As the background gas pressure is increased, shock waves and reduced-density ablation plumes become visible. Below 10 Torr, the ablation plume follows closely behind the shock wave. Between 20 and 100 Torr, the plume recedes behind the shock wave. Below 10 Torr and above about 200 Torr, both the plume and the shock expand with the same temporal power law dependence. Agreement is found between these power law dependences and those predicted by ideal blast wave theory. The DLRAP diagnostic clearly shows that the ablated material (CN molecule from polyimide and ground state neutral aluminum atoms from laser-ablated aluminum) resides in the ablation plume. $\mathrm{CN}$ molecules are detected in both argon and air environments proving that $\mathrm{CN}$ is generated as an ablation product and not by reaction with the background gas. As the background gas pressure and the time after ablation is increased, the film darkening due to the laser-ablated material begins to fade leaving only the nonresonant shadowgraphy component of the plume. The plume dynamics observed by DLRAP are discussed in terms of gas dynamics, plume chemical kinetics, material diffusion in the plume, and cluster/particulate formation.
\end{abstract}

\section{INTRODUCTION}

Laser ablation processing of materials has potential importance in micromachining for microelectronics applications such as the etching of photoresist and in the deposition of thin films of material. It has become increasingly clear that characterization of the hydrodynamics of ablated material and material heated by the ablation process is critical in the understanding of how thin films are deposited (e.g., chemical reactions, particulate content) or, for example, how an etched surface is affected by freshly ablated material (e.g., material redeposition).

The hydrodynamics of excimer laser ablation has been widely investigated by photoacoustic/photothermal techniques and by schlieren photography/shadowgraphy. Photoacoustic/photothermal techniques (piezoelectric probe or laser probe beam deflection technique) have been used to detect stress waves ${ }^{1}$ driven into the target and acoustic $^{2-4}$ and thermal waves ${ }^{2}$ driven into the background gas. The laser probe beam deflection technique has been used to estimate the mass inventory in ablation plumes expanding into vacuum. ${ }^{5}$ Shadowgraphy has permitted two-dimensional flow visualization showing shock/sound waves $^{6,7}$ and has also shown ablation plumes following the shock waves. ${ }^{8,9}$ Combining schlieren photography and the laser beam deflection technique revealed that the plumes

\footnotetext{
a) Present address: Department of Electrical and Computer Engineering, University of Illinois at Urbana-Champaign, 1406 W. Green Street, Urbana, IL 61801.
}

were actually reduced density cavities. ${ }^{8}$ The dark field imaging method has been used by Grun et al. ${ }^{10,11}$ to study the shock hydrodynamics from high-intensity $\left(3 \mathrm{TW} / \mathrm{cm}^{2}\right)$ $\mathrm{Nd}$ glass laser irradiation of aluminum and polystyrene.

Information on the dynamics of individual species transported from the ablated surface is of particular significance in understanding the chemical nature of freshly deposited films. Most investigations that have provided species-specific information on laser-ablation processing have been studies of emissive species mèasured by either emission spectroscopy or laser-induced fluorescence (LIF). The most complete species-resolved studies previously reported were the LIF studies by Dreyfus, Kelly, and Walkup ${ }^{12}$ on aluminum and $\mathrm{Al}_{2} \mathrm{O}_{3}$, and the LIF studies by Wang, Salzberg, and Weiner ${ }^{13}$ on aluminum. More recent LIF studies have been performed by Deshmukh, Rothe, and Reck ${ }^{14}$ on polymers. Another spatially resolved diagnostic was a combined streak-photography LIF experiment by Cappelli et al. ${ }^{15}$ Several previous investigations have directly probed nonemissive laser ablation products. One, a species-specific diagnostic, was a resonant ionization spectroscopy investigation of a nonemissive state of $\mathrm{CO}$ by Goodwin and Otis. ${ }^{16}$ Another was a Michelson interferometer study by Walkup, Jasinski, and Dreyfus ${ }^{17}$ of laserablated polyimide and alumina; that experiment, like laser beam deflection, ${ }^{5,8}$ was not species-specific. Absorption spectroscopy has been applied by Geohagen and Mashburn $^{18}$ and Cheung et al. ${ }^{19}$ to the study of laser-ablated material. Recently there have been mass spectroscopic (a 




FIG. 1. Experimental configuration.

nonoptical species-specific diagnostic) investigations of $\mathrm{KrF}$ laser ablation of $\mathrm{YBa}_{2} \mathrm{Cu}_{3} \mathrm{O}_{7-\delta}$ showing the influence of number of shots on the composition of the ablation plume. ${ }^{20,21}$

This investigation addresses the application of schlieren photography ${ }^{8}$ and a species-specific diagnostic, dye-laser resonance absorption photography (DLRAP), ${ }^{22}$ to the study of the hydrodynamics of excimer laser ablation processing of polymers and metals both in vacuum and background gas environments. Schlieren photography casts a shadow on film in response to the first spatial derivative of the density of one or more species parallel to the plane imaged on the film. In DLRAP, the light from an expanded, collimated laser beam absorbed by a plume of atomic or molecular species casts a shadow on photographic film. DLRAP combines the features of absorption spectroscopy and shadowgraphy. In absorption spectroscopy, the light at a particular wavelength is absorbed by a resonance transition of an atomic or molecular species. Conventional absorption spectroscopy uscs an arc lamp with a spectrometer and photomultiplier tube. In shadowgraphy, a shadow is cast on the film in response to the second derivative of the density due to one or more species; in DLRAP, the shadow is cast in response to the density of a single state of a selected species. Like the resonant diagnostics employed by Horioka et $a l^{23}$ and Mostovych et al. ${ }^{24}$ DLRAP is sensitive to any specific state, as long as it is strongly absorbing.

\section{EXPERIMENTAL CONFIGURATION}

The experimental configuration for DLRAP and Schlieren photography shown in Fig. 1 has been reported previously. ${ }^{8,22}$ Polyimide (Kapton and Vespel which are chemically identical), polyethyleneterephthalate (PET, mylar), and aluminum samples were ablated in argon, air, and nitrogen environments $\left(10^{-5}-760\right.$ Torr) by a $248 \mathrm{~nm}$ $\mathrm{KrF}$ excimer laser with a peak pulse energy of $1.5 \mathrm{~J}$ and a pulselength of $40 \mathrm{~ns}$. The hydrodynamic phenomena following the ablation event were diagnosed by means of Schlieren photography and dye laser resonance absorption photography (DLRAP) using a 20 ns pulselength $\mathrm{XeCl}$ excimer-pumped dye laser system. For DLRAP, the dye laser was operated with QUI dye allowing the laser to be tuned from approximately 380 to $400 \mathrm{~nm}$. The peak efficiency for this dye is $11 \%$ at $390 \mathrm{~nm}$. The laser line width is approximately $0.2 \mathrm{~cm}^{-1}$. The operating wavelength for DLRAP was tuned to the maximum absorption of the particular atomic or molecular transition that was being excited. For polyimide, we investigated the $388.34 \mathrm{~nm}$ absorption band head of $\mathrm{CN}$; for aluminum we investigated both the 394.4 and $396.1 \mathrm{~nm}$ absorption lines. Schlieren photography was performed using Rhodamine $6 \mathrm{G}$ dye with the dye laser tuned to $581 \mathrm{~nm}$. Schlieren and DLRAP measurements were recorded on type 47 Polaroid film. In all cases, it was necessary to insert neutral density (typically $N D=2$ ) filters into the path of the dye laser beam to prevent overexposure of the film. The neutral density filters were usually inserted between the camera and the ablation plume to attenuate the plasma light.

\section{EXPERIMENTAL RESULTS}

Figure 2 illustrates a series of resonant versus nonresonant dye-laser resonance absorption photographs for a variety of materials and environments to illustrate the versatility of the diagnostic. Figures 2 (a) and 2(b) illustrate DLRAP measurements of ground state neutral Al atoms present in an Al ablation plume expanding into vacuum. Figure 2(a) is taken with the dye laser tuned on-resonance to the $394.4 \mathrm{~nm}$ resonance and 2 (b) is taken with the dye laser tuned off resonance by $0.03 \mathrm{~nm}$. The delay between the dye laser and the excimer laser is $1 \mu \mathrm{s}$. Note that the $\mathrm{Al}$ plume is undetectable in the nonresonant case but almost completely fills the photograph in the resonant case. The white glow at the surface is probably symptomatic of a laser-produced plasma at the surface as it was eliminated by locating neutral density between the plume and the camera. Figures 2(c) and 2(d) are also measurements of the neutral aluminum atom ablation plume of an Al sample but the background is 250 Torr Ar instead of vacuum. Figure 2(c) is tuned to the $394.4 \mathrm{~nm}$ line and 2(d) is tuned $0.5 \mathrm{~nm}$ off-resonance. The delay between the $\mathrm{KrF}$ laser and the dye laser is $50 \mu$ s for this case. Figure 2(c) illustrates that the ablation products travel in the ablation plume. In the resonant case, the ablation plume is completely blackened but in the nonresonant case the plume is barely visible. This shows that the darkening on the photographs is not caused by particulate from the ablation of the aluminum target. Note also that at times on the order of $50 \mu \mathrm{s}$, the darkened region corresponding to ablation products is still in contact with the surface. It should be noted that the shock wave is visible by shadowgraphy in the nonresonant data of Fig. 2(d). Figures 2(e) and 2(f) illustrate the use of the DLRAP diagnostic on aluminum in vacuum at a different wavelength at a delay time of $200 \mathrm{~ns}$; Fig. 2(e) is tuned to the $396.1 \mathrm{~nm}$ resonance line and Fig. 2(f) is 0.06 nm off-resonance. DLRAP measurements were also illustrated with other materials: iron and polyimide. Figures $2(\mathrm{~g})$ and $2(\mathrm{~h})$ demonstrate resonance at the $385.99 \mathrm{~nm}$ neutral iron line and off-resonance, $\Delta \lambda=0.01 \mathrm{~nm}$, for 




FIG. 2. On- and off-resonance dye laser resonance absorption photographs. Al in vacuum $1 \mu \mathrm{s}$, (a) $394.4 \mathrm{~nm}$ line on-resonance, (b) $\Delta \lambda=0.03 \mathrm{~nm}$ off-resonance; Al in 250 Torr Ar $47 \mu \mathrm{s}$, (c) $394.4 \mathrm{~nm}$ line on-resonance, (d) $\Delta \lambda=0.5 \mathrm{~nm}$ off-resonance; Al in vacuum $200 \mathrm{~ns}$, (e) $396.1 \mathrm{~nm}$ line on-resonance, (f) $\Delta \lambda=0.06 \mathrm{~nm}$ off-resonance; $\mathrm{Fe}$ in vacuum $200 \mathrm{~ns},(\mathrm{~g}) 385.99 \mathrm{~nm}$ line on-resonance, (h) $\Delta \lambda=0.01 \mathrm{~nm}$ off-resonance; $\mathrm{CN}$ from polyimide in vacuum, $200 \mathrm{~ns}$, (i) $388.34 \mathrm{~nm}$ line on-resonance, (j) $\Delta \lambda=1.6 \mathrm{~nm}$ (to the red) off-resonance:

DLRAP measurements on iron ablation plumes $200 \mathrm{~ns}$ after the laser pulse. Molecular absorption was also demonstrated by ablating $\mathrm{CN}$ molecules from polyimide. Figures 2(i) and 2(j) illustrate polyimide ablation plumes expanding into vacuum $200 \mathrm{~ns}$ after the ablating $\mathrm{KrF}$ laser pulse. Figure 2(i) is tuned to the $\mathrm{CN}$ absorption band head at $388.34 \mathrm{~nm}$ and $2(\mathrm{j})$ is the off-resonance condition at $\Delta \lambda=1.6 \mathrm{~nm}$ to the red. Since polymers are more easily ablated than metals (the threshold for polyimide is $25 \mathrm{~mJ} /$ $\mathrm{cm}^{2}$ and the threshold for metals are in excess of $1 \mathrm{~J} / \mathrm{cm}^{2}$ ), ablated material is weakly visible for expansion into vacuum for the nonresonant case because of the substantial density gradients caused by the ablated material.

\section{A. Vacuum measurements}

Dye laser resonance absorption photographic measurements of ablation hydrodynamics in vacuum were per- formed on aluminum and on polyimide (Kapton and Vespel). Figure 3 presents the temporal evolution of a polyimide (Kapton) ablation plume expanding into vacuum; the fluence for these measurements was $18 \mathrm{~J} / \mathrm{cm}^{2}$. The dye laser was tuned to the $\mathrm{CN}$ absorption band head at $388.34 \mathrm{~nm}$. and the delay between the dye laser and the $\mathrm{KrF}$ excimer laser was (a) 100 , (b) 320 , (c) 540 , and (d) 820 ns. As for the case of expansion of neutral aluminum into vacuum, the $\mathrm{CN}$ plume shows expansion in both the perpendicular and parallel directions with respect to the target surface. The expansion of $\mathrm{CN}$ into vacuum normal to the target surface is much more pronounced than the expansion parallel to the target surface. This is a markedly different behavior than is displayed by the expanding neutral aluminum plume ${ }^{22}$ in which perpendicular and parallel expansion velocities are similar. 



FIG. 3. Dye laser resonance absorption photographs (CN $388.34 \mathrm{~nm}$ line) of ablation plumes expanding into vacuum generated by $18 \mathrm{~J} / \mathrm{cm}^{2}$ of $\mathrm{KrF}$ excimer laser radiation incident on polyimide. The delay between the dye laser pulse (DLRAP) and the $\mathrm{KrF}$ excimer laser pulse was (a) 100 , (b) 320 , (c) 540 , (d) 820 ns.

A linear relationship was observed for the position of the leading edge of the visible part of the ablation plume on the photograph for both Al and CN. Figure 4(a) shows the $\mathrm{CN}$ plume edge position as a function of time for fluences between 4 and $18 \mathrm{~J} / \mathrm{cm}^{2}$. Figure $4(\mathrm{~b})$ shows the corresponding expansion velocities as a function of fluence. The CN expansion velocities were slower than those of $\mathrm{Al}$ by about a factor of 4 . The previously reported expansion velocities of Al varied between 0.5 to $3.3 \mathrm{~cm} / \mu$ s for fluences between 1.5 and $7 \mathrm{~J} / \mathrm{cm}^{2}$.

An important observation is that the darkest region of the photographs is always near the surface. Further, this region remains dark for times much longer than the laser pulse and the near-surface image is the last to fade away. This means that some of the ablated material remains near the surface for times much longer than the ablating laser pulse. For the case of laser-ablated $\mathrm{CN}$ (from polyimide) and $\mathrm{Al}$ expanding into vacuum, material is seen to remain near the surface longer than $1 \mu \mathrm{s}$. This sort of behavior is consistent with the reflected rarefaction wave model described in Refs. 5 and 25.

For polymers in the off-resonance case, we observe jetlike structures at the edge of the ablation regions. This jetting, observed by Gabl et al. ${ }^{26}$ at much higher fluences, is probably due to fluence nonuniformities in the $\mathrm{KrF}$ laser spot. It is likely that the material ablated at the edges is much cooler and expands at a slower rate than the material ablated at the center of the laser spot. The ablated polymeric material at the edges is probably also of a higher molecular weight than the material in the middle. There could then be a shear between the two flow regimes creating a large density gradient between the two regions. The cold and high molecular weight edge could also act as a wall or nozzle directing the ablated material forward.


FIG. 4. (a) Position of $\mathrm{CN}$ (from polyimide) plume edge measured by dye laser resonance absorption photography as a function of time after the excimer laser pulse for different excimer laser fluences: $(0) 18 \mathrm{~J} / \mathrm{cm}^{2}$, (+) $10 \mathrm{~J} / \mathrm{cm}^{2}$, (A) $7 \mathrm{~J} / \mathrm{cm}^{2}$, (E) $4 \mathrm{~J} / \mathrm{cm}^{2}$. (b) Expansion velocities of $\mathrm{CN}$ plumes perpendicular to the surface, as a function of excimer laser fluence. These velocities were obtained by taking the slope of the data presented in (a).

\section{B. Experiments in gas backgrounds}

To gain insight into the nature of the material ablated from polymers, experiments were conducted on PET in air, $\mathrm{N}_{2}$, and Ar from mTorr pressures to atmospheric pressure environments. Figure 5 presents Schlieren photographs of laser ablation generated plumes in atmospheric pressure air and $\mathrm{N}_{2}$ at $2 \mathrm{~J} / \mathrm{cm}^{2}$ for two different delay times: 250 and $500 \mu \mathrm{s}$. Note that for both delay times, the plume in air is larger and smoother than that in $\mathrm{N}_{2}$ only. For plumes in Ar between 1 and $4 \mathrm{~J} / \mathrm{cm}^{2}$, the behavior is the same as that predicted for $\mathrm{N}_{2}$. For this fluence regime, it was found that the size of the plume (radius or the plume length) in air was always $20 \%-30 \%$ larger than the plume created with the same fluence in $\mathrm{N}_{2}$ or Ar. This suggests that the heated ambient gas and ablated material mixture is undergoing a chemical reaction and may generate a turbulent diffusion flame. ${ }^{27}$ If combustion were to occur, it would take place in the plume within which DLRAP measurements of the type presented in Figs. 2(c) and 2(d) show that the ablation products reside. Laser beam deflection measurements and Schlieren photography have been used to perform an energy balance on the plume. ${ }^{8}$ Those measurements showed that the energy contribution from the combustion of the ablated polymer could explain the increase in the size of the plumes in air versus other gases.

DLRAP measurements were performed on both alu- 
(a)


(b)


(d)

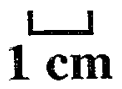

FIG. 5. Schlieren photographs comparing $\mathrm{KrF}$ laser-ablation generated plumes from PET in $\mathrm{N}_{2},(\mathrm{a}, \mathrm{c})$ and air $(\mathrm{b}, \mathrm{d})$. The delay between the dye laser pulse (Schlieren) and the $\mathrm{KrF}$ excimer laser is $250 \mu \mathrm{s}$ for (a) and (b) and $500 \mu \bar{s}$ for (c) and (d). The $\mathrm{KrF}$ excimer laser fluence was 2 $\mathrm{J} / \mathrm{cm}^{2}$.

minum (99.99\% pure, Johnson Matthey) and CN (from laser-ablated Vespel) plumes as a function of time after the $\mathrm{KrF}$ laser pulse and background gas pressure for both air and argon environments. Figure 6 presents the dynamics of

(a)


(b)

(c)


(d)

(e)


(f)

FIG. 6. Dye laser resonance absorption photographs of $5 \mathrm{~J} / \mathrm{cm}^{2} \mathrm{KrF}$ laser-ablated polyimide (Vespel) in air from 8.8 to 700 Torr $4 \mu$ s after $\mathrm{KrF}$ laser pulse: (a) 8.8 , (b) 22 , (c) 50 , (d) 150 , (c) 300 , (f) 700 Torr. (a)


(c)



(b)
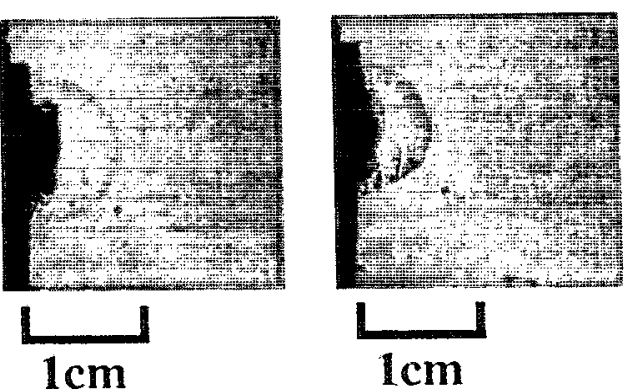

(f)

FIG. 7. Dye laser resonance absorption photographs of $5 \mathrm{~J} / \mathrm{cm}^{2} \mathrm{KrF}$ laser-ablated polyimide (Vespel) in argon from 9.6 to 700 Torr $4 \mu$ s after $\mathrm{KrF}$ laser pulse: (a) 9.6 , (b) 36 , (c) 75 , (d) 150 , (e) 300 , and (f) 700 Torr.

the $\mathrm{CN}$ ablation plume from laser-ablated $\left(5 \mathrm{~J} / \mathrm{cm}^{2}\right.$ at 248 $\mathrm{nm})$ Vespel in air between 8.8 and 700 Torr at $4 \mu$ s after the $\mathrm{KrF}$ laser pulse. The dye laser was tuned to the $\mathrm{CN}$ band head at $388.34 \mathrm{~nm}$. Figure 7 shows the plume dynamics of $\mathrm{CN}$ produced from $5 \mathrm{~J} / \mathrm{cm}^{2} \mathrm{KrF}$ laser-ablated Vespel at the same fluence in 9.6 to 700 Torr of argon at 4 $\mu$ s after the $\mathrm{KrF}$ laser pulse. The plume is seen to closely follow the weak shock at low pressures (less than 20 Torr). Note also that the shocks are not perfectly spherical but are more oblong. Particularly in Fig. 6(a) and Fig. 7(a), the shocks can be seen to have profiles that are molded to the irregularly profiled ablation plumes. This lends further weight to the argument that the plume acts like a piston which drives the laser-ablation generated shock waves. ${ }^{8}$ We believe that these irregular plume profiles are caused by nonuniformities in the KrF laser spot on the Vespel sample. At background gas pressures greater than approximately 20 Torr, the $\mathrm{CN}$ ablation plume tends to lag behind the shock; the shock plume gap expands from less than 1 $\mathrm{mm}$ to distances on the order of $7 \mathrm{~mm}$. At 700 Torr, the plume, compressed by the background gas, moves barely 1 $\mathrm{mm}$ off the surface. Note also that between 20 and 50 Torr, the plume front becomes less filamentary, the protruding plume fingers having disappeared.

Plots of the shock-CN plume dynamics from $\mathrm{KrF}$ laser-ablated Vespel DLRAP measurements are presented in Fig. 8(a) for air and Fig. 8(b) for argon. An important 



Pressure (Torr)

FIG. 8. Shock and plume position as a function of background gas pressure $4 \mu \mathrm{s}$ after the $\mathrm{KrF}$ excimer laser pulse measured by dye laser resonance absorption photography (CN $388.34 \mathrm{~nm}$ line) for $5 \mathrm{~J} / \mathrm{cm}^{2} \mathrm{KrF}$ laser-ablated polyimide (Vespel) in (a) air and (b) argon environments: region A, plume closely follows shock; region B, "kink" in which plume recedes from shock; region $\mathrm{C}$, plume and shock separate but follow the same power law dependence.

observation is that for all time delays where the plume is visible, a "kink" occurs in the plume position versus pressure curve at approximately 20 Torr. At lower pressures, the plume trails closely behind the shock. At pressures above 100 Torr the plume trails far behind the shock but expands according to the same power law (as a function of pressure) as the shock.

As the delay time between the dye laser diagnostic pulse and the $\mathrm{KrF}$ laser-ablation pulse was increased, the $\mathrm{CN}$ resonance absorption image appeared to decay so that the DLRAP photos tended towards nonresonant shadowgraphs. The decay of the $\mathrm{CN}$ in the plume as a function of time can be illustrated best by the time study of the shockplume dynamics in 250 Torr of argon presented in the DLRAP photographs of Fig. 9. The $\mathrm{KrF}$ laser fluence for these measurements was $5.7 \mathrm{~J} / \mathrm{cm}^{2}$. Figures 9 (a) and 9 (b) are the off-resonance (a) and on-resonance (b) DLRAP cases for the shock/plume dynamics $8.8 \mu$ s after the laser pulse. It should be noted that when the dyc laser is tuned off-resonance, the diagnostic photographs represent shadowgraphy images of gas dynamics and shock waves. In Fig. 9 (c) at $0.8 \mu$ s delay, the plume is directly behind the shock. This is identical to the phenomena observed for later times at lower pressures [e.g., Fig. 7(a) or Fig. 8(a)]. By $3.8 \mu$ s, Fig. 9(d), the plume has lagged behind the shock. The plume and shock continue to expand until be- (a)
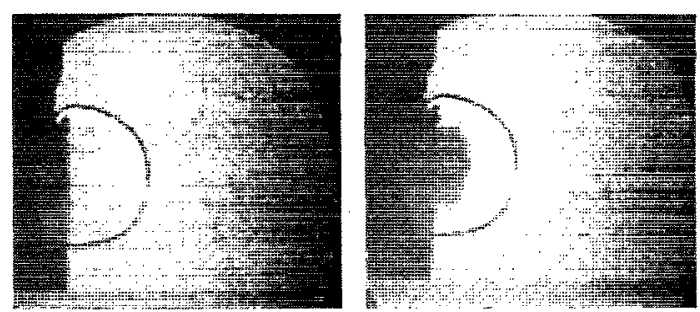

(b)


(d)

(e)


(f) (g)
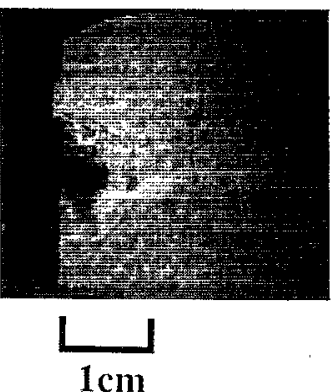



(h)



FIG. 9. Dye laser resonance absorption photographs (CN $388.34 \mathrm{~nm}$ line) photographs of ablation plumes expanding into 250 Torr of argon generated by $5.7 \mathrm{~J} / \mathrm{cm}^{2}$ of $\mathrm{KrF}$ excimer laser radiation incident on polyimide (Vespel). The delay between the dye laser pulse (DLRAP) and the $\mathrm{KrF}$ excimer laser pulse was (a) $8.8 \mu$ s (off-resonance, $\Delta \lambda=0.6 \mathrm{~nm}$ ), (b). $8.8 \mu \mathrm{s}$ (on-resonance), (c) $0.8 \mu \mathrm{s}$, (d) $3.8 \mu \mathrm{s}$, (e) $18.8 \mu \mathrm{s}$, (f) $53.8 \mu \mathrm{s}$, (g) $83.8 \mu \mathrm{s}$, (h) $193.8 \mu \mathrm{s}$, and (i) $993.8 \mu \mathrm{s}$.

tween 53.8 and $83.8 \mu$ s, Figs. $9(\mathrm{f})$ and $9(\mathrm{~g})$, the plume begins to fade. At times greater than this, the shadowgraphy images show the evolution of a "mushroom cloud" that is fully developed by $1 \mathrm{~ms}$. This mushroom cloud has been observed previously by our schlieren photography diagnostic. ${ }^{8}$ After $50 \mu$ s, however, $\mathrm{CN}$ is no longer resonantly detected by the DLRAP diagnostic. A plot of the temporal $\mathrm{CN}$ plume/shock evolution is presented in Fig. 10, which shows the decoupling of the plume and shock at times greater than $20 \mu \mathrm{s}$. 




FIG. 10. Temporal evolution of shock $(x)$ and plume $(+=$ DLRAP, - =shadowgraph) position measured by dye laser resonance absorption photography (CN $388.34 \mathrm{~nm}$ line) for $5.7 \mathrm{~J} / \mathrm{cm}^{2} \mathrm{KrF}$ laser-ablated polyimide (Vespel) in 250 Torr of argon. A indicates the shock wave, B indicates the region in which the shock and plume travel with the same power law dependence and the plume is completely darkened, $C$ indicates the temporal evolution of the plume as detected by shadowgraphy, i.e., nondarkened, and D indicates the temporal evolution of the DLRAP plume as it recedes (or fades) behind the plume evidenced by shadowgraphy.

An interesting additional observation is that the $\mathrm{CN}$ appears similarly in DLRAP measurements in both argon and air background gas environments. This implies that $\mathrm{CN}$ is generated by the ablation process and is not a reaction product from an interaction between the background gas and the ablated material. $\mathrm{CN}$ is instead a decomposition product of the ablated material. When exposed to UV light, polymers tend to first liberate $\mathrm{CO}$ groups by Norrish's reactions. ${ }^{28}$ This implies that the $\mathrm{CN}$ detaches from the adjacent benzene ring in a subsequent step. ${ }^{28,29}$

Similar measurements were performed for $\mathrm{KrF}$ laserablated aluminum in both air and argon backgrounds. Figure 11 shows dye laser resonance absorption photographs of $\mathrm{KrF}$ laser-ablation generated aluminum neutral atom plumes with shock waves in an air background at various pressures at a delay of $2.28 \mu \mathrm{s}$ after the $6.1 \mathrm{~J} / \mathrm{cm}^{2} \mathrm{KrF}$ laser pulse. The same phenomena observed for $\mathrm{CN}$ molecules from Vespel has been observed for aluminum neutral atoms. In Figs. 11(a) and $11(\mathrm{~b})$ at 5.6 and 13 Torr, the plume is essentially attached to the shock structure. At 50 Torr, Fig. 11(d), the shock and Al neutral atom plume have separated. Note that in Fig. 11(c) at 35 Torr and 11 (d) at 50 Torr, filaments protrude from the Al plume. Again, these filaments are believed to be caused by nonuniformities in the $\mathrm{KrF}$ laser spot on the aluminum target surface. Also displayed in these photographs are slightly deformed shock waves. They do not follow the filaments as perfectly as in the case of $\mathrm{CN}$ at low pressures. Indeed the shock tends to travel fastest where the Al plume is closest to the metal surface, in a sense opposite to the piston model. In Figs. 11(e) and $11(\mathrm{f})$ at 200 and 700 Torr, the aluminum plume is compressed close to the target surface. Still observable, however, is the irregular shock wave structure.

Plots of the aluminum neutral atom plume and shock position versus pressure measurements of Fig. 11 are sum-


(b)


(d)


$(1)$


FIG. 11. Dye laser resonance absorption (A1 $394.4 \mathrm{~nm}$ line) photographs of $6.1 \mathrm{~J} / \mathrm{cm}^{2} \mathrm{KrF}$ laser-ablated aluminum in air from 5.6 to 700 Torr 2.3 $\mu$ s after KrF laser pulse: (a) 5.6, (b) 13 , (c) 35 , (d) 50 , (e) 200, and (f) 700 Torr.

marized in Fig. 12(a) and, for the case of $\mathrm{Al}$ ablated into an argon environment, Fig. 12(b). As for the case of $\mathrm{CN}$ molecules DLRAP measurements from laser-ablated polyimide, we still observe the kink in the plume position versus pressure between 20 and 100 Torr.

The temporal evolution of the aluminum neutral atom ablation plume was measured by DLRAP for 35 Torr of argon and is presented in Figs. 13 and 14. Features similar to those presented for the temporal evolution of the $\mathrm{CN}$ molecule plume are observed: the shock following the plume at early times (less than $1 \mu \mathrm{s}$ ) and the recession of the plume behind the shock at later times. Although not presented in this figure, erosion of the plume, i.e., a fading of the Al neutral atom DLRAP image to a nonresonant shadowgraph, is observed.

\section{DISCUSSION}

\section{A. Plume gas dynamics}

A wide range of gas-dynamic phenomena are observable with the DLRAP diagnostic. In the background gas environments shocks and reduced density plumes are detected. As the background gas pressure is decreased, DLRAP clearly shows ablated material present in the ablation plume. The DLRAP image of the ablation plume is completely darkened by the ablated material at pressures on the order of 10 Torr. At this pressure, the plume begins to follow immediately behind the shock. The shock image 



FIG. 12. Shock wave $(x)$ and plume $(+)$ position from DLRAP (Al $394.4 \mathrm{~nm}$ line) $2.3 \mu \mathrm{s}$ after a $6.1 \mathrm{~J} / \mathrm{cm}^{2} \mathrm{KrF}$ laser pulse incident on an aluminum target as a function of background gas pressure: (a) air, (b) argon.

also becomes less sharp at lower pressures. As the background gas pressure lowers to the $10^{-5}$ Torr scale, a DLRAP image ${ }^{22}$ characteristic of a reflected rarefaction wave is displayed. ${ }^{5,22}$

The shock/plume position versus pressure and time relationships tend to follow spherical blast wave scaling ${ }^{30}$ shown in Eq. (1),

$$
R \propto\left(\frac{E}{\rho_{0}}\right)^{1 / 5} t^{2 / 5} \propto\left(\frac{E}{P}\right)^{1 / 5} t^{2 / 5},
$$

where $R$ is the shock radius, $E$ is the energy that drives the shock, $P$ is the pressure, and $t$ is the time. The shocks for both polyimide (Fig. 8) and aluminum (Fig. 12) ablation follow these pressure and time scalings almost exactly. Temporally, the aluminum ablation-induced shock (Fig. 14,35 Torr, argon) exactly follows a $t^{0.4}$ relationship. The temporal scaling of the polyimide (Fig. 10, 250 Torr, argon) shock deviates slightly with a $t^{0.5}$ relationship. The temporal evolution for shocks at $10 \mathrm{~J} / \mathrm{cm}^{2}$ for $\mathrm{KrF}$ excimer laser ablation of PET in atmospheric air was previously found to be proportional to $t^{0.7}$ which agrees less well with the ideal blast wave scaling. ${ }^{8}$ This is probably because the ideal blast wave model is more valid for lower background gas pressures, all other parameters being equal.



FIG. 13. Dye laser resonance absorption (Al $394.4 \mathrm{~nm}$ line) photographs of ablation plumes expanding into 35 Torr of argon generated by 6.9 $\mathrm{J} / \mathrm{cm}^{2}$ of $\mathrm{KrF}$ excimer laser radiation incident on aluminum. The delay between the dye laser pulse (DLRAP) and the $\mathrm{KrF}$ excimer laser pulse was (a) $220 \mathrm{~ns}$, (b) $560 \mathrm{~ns}$, (c) $1.28 \mu \mathrm{s}$, (d) $1.76 \mu \mathrm{s}$, (e) $3.76 \mu \mathrm{s}$, and (f) $5.84 \mu \mathrm{s}$.

This spherical blast wave scaling, $P^{-0.2}$, applies to the plume as well as the blast waves except in the region of the "kink." For polyimide ablation, as presented in Figs. 8(a) and $8(\mathrm{~b})$, the plume fronts scale as $P^{-0.6}$ in argon and $P^{-0.8}$ in air; for aluminum ablation, as presented in Figs. $12(\mathrm{a})$ and $12(\mathrm{~b})$, the plume fronts scale as $P^{-0.6}$ in argon and $P^{-0.7}$ in air.

A number of other physical proccsses can occur in the ablation plume-gas dynamics observable with DLRAP:

(1) Oxidation/chemical kinetics: plume material with



FIG. 14. Temporal evolution of $6.9 \mathrm{~J} / \mathrm{cm}^{2} \mathrm{KrF}$ laser-ablated aluminum generated shock-wave $(X)$ and plume $(+)$ in a 35 Torr argon background measured by DLRAP (Al $394.4 \mathrm{~nm}$ line). 
background gas, plume material with plume material (in both background gas or vacuum environments);

(2) Material and or thermal diffusion (in background gas); and

(3) Particulate formation/polymerization while the plume cools (in both background gas or vacuum environments).

\section{B. Oxidation/chemical kinetics}

The chemical kinetics of ablated species, both in vacuum and gas backgrounds, is complex primarily because the exact nature of the species present in a laser-ablated plume is not well characterized at times within microseconds after the ablating laser pulse. Thermodynamic conditions vary widely from that of a critical fluid (high pressure and temperature) at the time of ablation to a rarefied gas with a frozen velocity distribution at times long after the ablation event. For polymers, it is known that ablation can result in the production of polymer fragments of various masses to free atomic species. There exist many possible reaction pathways even for the much more simple system of laser-ablated aluminum in an oxygen environment. ${ }^{31}$ What reaction rates are available are only measured for very limited temperature and pressure regimes.

DLRAP measurements (e.g., Fig. 9) show both a fading of the darkening of the ablation plume with increasing time and a recession of the plume from the shock front that is observable for both polymers and metals and in both inert and reactive background gases. One possible explanation for these effects is that the fading and recession are caused by a depletion of resonance absorbers through reactions with the background gas. However, data suggest that chemical kinetics does not play an important role in plume dynamics since similar behavior is observed in both inert and reacting background gases and unoxidized aluminum is present on the order of microseconds in 50-200 Torr of air. This is contrary to what one would expect given the high reaction rate for aluminum oxidation. ${ }^{31}$ The presence of unoxidized Al in the plume at long times suggests either that the depletion of $\mathrm{Al}$ in the background gas or propagation of $\mathrm{Al}$ into the background gas may be controlled by another mechanism: diffusion (for propagation) or cluster formation (for depletion).

A hypothesis that would explain why chemical kinetics appears not to radically affect plume gasdynamics observable by DLRAP is that the initial speed of the aluminum particles into the background gas is high enough to increase the reaction mean free path such that the gas dynamics are dominated by diffusion or cluster formation. Reactions between species characterized by two Maxwellian distributions at the same temperature having reaction rates, $k$, that are independent of temperature, possess cross sections, $\sigma$, that are inversely proportional to center of mass particle velocity or $k / v_{\text {com. }}$. The reaction rate for the reaction between molecular oxygen and neutral aluminum is $3 \times 10^{-11} \mathrm{~cm}^{3} /$ (molecule $\mathrm{s}$ ) and is independent of temperature. If we assume that the ablation plume can be modeled as a beam of monoenergetic particles with a velocity, $v_{\text {com }}$, entering a still gas with temperature, $T_{\text {gas }}$, the



FIG. 15. Plot of the mean free path as a function of background gas (air) pressure for a beam of noninteracting neutral $\mathrm{Al}$ atoms with an initial velocity of $4500 \mathrm{~m} / \mathrm{s}$ in a background gas at 300 and $4000 \mathrm{~K}$. The main interaction between the beam and the background gas is $\mathrm{AI}+\mathrm{O}_{2} \rightarrow \mathrm{AlO}$ $+\mathrm{O}$ and the cross section is derived from the data of Mann and Weaver (Ref. 29).

reaction rate of beam particles with the background gas is independent of the background gas temperature and is simply

$$
\begin{aligned}
& \frac{d n_{\text {beam }}}{d t}=n_{\text {beam }} n_{\text {gas }}\left\langle\sigma v_{\text {com }}\right\rangle, \\
& \frac{d n_{\text {beam }}}{d t}=n_{\text {beam }} n_{\text {gas }} k .
\end{aligned}
$$

Therefore, the time for the beam density to fall off by $1 / e$ is $1 /\left(n_{\mathrm{gas}} k\right)$. The distance for the beam to persist to a $1 / e$ density would then be $v_{\text {beam }} /\left(n_{\text {gas }} k\right)$. A plot showing the mean free path of an aluminum particle (with a velocity of $4500 \mathrm{~m} / \mathrm{s})^{22}$ in two different air environments (300 and $4000 \mathrm{~K})^{30}$ is presented in Fig. 15. Assuming that the controlling mechanism for aluminum loss due to oxidation is its reaction with molecular oxygen, increasing the background gas temperature from 300 to $4000 \mathrm{~K}$ increases the pressure at which the $1 \mathrm{~cm}$ mean free path occurs from less than 1 Torr to above 20 Torr. This is because of the dissociation of the oxygen molecule.

\section{Diffusion}

Because nearly the same $\mathrm{CN}$ and $\mathrm{Al}$ plume behavior is observed, with respect to the darkening observed in DLRAP, both in Ar and air backgrounds, we must investigate phenomena that could explain it that are not significantly dependent on the background gas.

A possible mode of interaction between the plume and the background gas is by material diffusion, i.e., the diffusion of hot noninteracting particles in the cold background gas. The darkening of the plumes would, in light of this model, be indicative of how far the plume has propagated by diffusion.

Most mass diffusion coefficients are approximately 1 $\times 10^{-5} \mathrm{~m}^{2} / \mathrm{s}$ at $300 \mathrm{~K}$ and $1 \mathrm{~atm}$ and scale roughly as $T^{3 / 2}$ and $1 / P$ as shown in Eq. (4), ${ }^{32}$ 


$$
D_{A B}=\frac{\left[1.084-0.249 \sqrt{\left(1 / M_{A}\right)+\left(1 / M_{B}\right)}\right] T^{3 / 2} \sqrt{\left(1 / M_{A}\right)+\left(1 / M_{B}\right)}}{P\left(r_{A B}\right)^{2} f} \times 10^{-4},
$$

where $D_{A B}$ is the mass diffusion coefficient for particle $A$ diffusing in gas $B$ in units of $\mathrm{m}^{2} / \mathrm{s}$, and $M_{A}$ and $M_{B}$ are the molecular weights of $A$ and $B$, respectively. $T$ is the gas temperature (same for $A$ and $B$ ), $P$ is the pressure, $r_{A B}$ is the molecular separation at collision, and $f$ is the collision function (found in Ref. 32). The diffusion length of noninteracting particles in a gas can be written as

$$
D(T, P)=x^{2} / t,
$$

where $x$ is the diffusion length, $t$ is the time, and $D$ is the mass diffusion coefficient. The plume position would then be proportional to $P^{-0.5}$. The experimental plumes tend to follow the $P^{-0.2}$ power law except in the "kink" regions between 20 and 100 Torr where they are observed to follow $P^{-0.6}$ to $P^{-0.8}$. Figure 16 shows a fit of the expression for diffusion length to the plume position versus pressure plot for $\mathrm{Al}$ in an air background at $4 \mu$ s for various temperatures. Overall, the data can be modeled satisfactorily as diffusing particles in the range of 3-10 eV.

\section{Cluster or particulate formation}

Removal of ground state $\mathrm{Al}$ in argon could also be explained by the formation of an excimer or by bonding between $\mathrm{Al}$ atoms leading ultimately to cluster or particulate formation. Particulate is visible to the eye in both $\mathrm{Al}$ and polyimide ablation by scattering of $\mathrm{HeNe}$ laser light. Sappey and Gamble ${ }^{33}$ in their copper ablation experiments have spectroscopically shown the presence of the copper dimer, $\mathrm{Cu}_{2}$, the first step in the formation of a copper cluster. They also indicated that the background gas, in their case $\mathrm{He}$, is important in providing a third body to promote the formation of the dimer. Experiments and simulations have been performed by Yamada et al. ${ }^{34}$ on alu-



FIG. 16. Estimated diffusion length as a function of pressure for $\mathrm{Al}$ atoms in a background gas in which the $\mathrm{Al}$ atoms and the background gas are assumed to be at the same temperature. A typical diffusion coefficient at $300 \mathrm{~K}, 760$ Torr of $1 \times 10^{-5} \mathrm{~m}^{2} / \mathrm{s}$ (Ref. 32) is used. A corresponds to 10 $\mathrm{eV}, \mathrm{B}$ corresponds to $7 \mathrm{eV}, \mathrm{C}$ corresponds to $5 \mathrm{eV}$, and $\mathrm{D}$ corresponds to $3 \mathrm{eV}$. minum cluster formation using a steady-state heated crucible-nozzle system. If, in the expanding aluminum plume, a condition of a supersaturated aluminum vapor exists, then, according to Yamada's work, cluster formation would be expected. To promote cluster formation, the temperature of the vapor must be lowered and/or its pressure increased. Therefore, a supersaturated vapor could be produced at the moment of ablation (at $T_{\text {vapor }}, P_{\text {plume }}$ is greater than the equilibrium vapor pressure) or could be achieved by rapid cooling of the vapor without a rapid drop in number density by mixing the vapor with cold background gas ( $T_{\text {vapor }}$ is lowered, lowering the equilibrium vapor pressure below $P_{\text {plume }}$ ). In the latter case, we would expect the particulate or cluster formation (and hence DLRAP plume erosion) to occur at the plumebackground gas interface and recede backwards (towards the target surface).

A model proposed by Rayleigh ${ }^{35}$ for laser-guided discharge channels in which cold gas outside the channel is injected into the channel by turbulent mixing at the channel-background gas interface would be expected to result in such a phenomenon. We observe such an erosion for the case of aluminum in Figs. 11(c) and 11(d). The background gas pressure of 20 Torr could be a critical pressure at which the ablated vapor becomes severely supersaturated, explaining the "kink" in the DLRAP plume position versus pressure curves.

Kuper and Brannon have recently investigated deposited debris from $\mathrm{KrF}$ laser ablation of polyimide as a function of background gas pressure. They found that the debris scaled as $P^{-1 / 3}$ for a variety of gases. They also hypothesized a relationship between shock/plume hydrodynamics and particulate generation. ${ }^{36}$

\section{v. CONCLUSIONS}

Schlieren photography and dye laser resonance absorption photography (DLRAP) have been shown to be effective diagnostics for the study of the dynamics of laser ablation processing of polymers and metals. In background gases, schlieren photography clearly shows sound waves, shock waves, and reduced density plumes traveling from the target surface. The scaling of the shock positions with background gas pressure and time have been compared to ideal blast wave scaling and found to be in excellent agreement. The dynamics of the expansion of ablated material into vacuum and reduced density ablation plume into background gases were probed with DLRAP. In vacuum, the ablation plumes were observed to expand like reflected rarefaction waves. In background gases, DLRAP showed that the ablated material resides in the ablation plume. This resonant diagnostic also showed in the case of laser ablated polyimide, that the $\mathrm{CN}$ molecules measured were an ablation product and not formed by a chemical inter- 
action between the background gas and ablation products. The ablation plumes tended to follow the same blast wave scaling as did the shock waves except in the region of 20-100 Torr. The resonant features of the ablation plumes were found to be similar in inert and reactive gas backgrounds. Particularly, the darkening from $\mathrm{CN}$ and ground state neutral aluminum atoms in various gases were observed to fade similarly, both temporally and as a function of background gas pressure. Several hypotheses have been proposed to explain the behavior of laser ablated matter in gas backgrounds. Gas dynamics play a major role, and chemical kinetics effects do not appear to play a significant role in plume behavior in gas environments. The possible influence of physical mechanisms such as diffusion and cluster formation appear more likely. To conclusively argue that a depletion of absorbers observed by DLRAP is due to a specific mechanism requires a measurement of the density of the species that has not been presented in this paper. Further experiments are underway to determine which mechanisms are important in laser ablation.

\section{ACKNOWLEDGMENTS}

This research has been supported by a grant from the National Science Foundation (CTS-9108971). The authors wish to thank R. Spears for technical assistance. RAL has a DOE Magnetic Fusion Energy Technology fellowship.

${ }^{1}$ P. E. Dyer and R. Srinivasan, Appl. Phys. Lett. 48, 445 (1986).

${ }^{2}$ J. A. Sell, D. M. Heffelfinger, P. L. G. Ventzek, and R. M. Gilgenbach, J. Appl. Phys. 69, 1330 (1991).

${ }^{3}$ E. Hunger, H. Pietsch, S. Petzoldt, and E. Matthias, Appl. Surf. Sci. (in press).

${ }^{4}$ W. P. Leung and A. C. Tam, Appl. Phys. Lett. 60, 23 (1992).

${ }^{5}$ P. L. G. Ventzek, R. M. Gilgenbach, D. M. Heffelfinger, and J. A. Sell, J. Appl. Phys. 70, 587 (1991).

${ }^{6}$ R. Srinivasan, B. Braren, K. Casey, and M. Yeh, Appl. Phys. Lett. 55, 2790 (1989).

${ }^{7}$ R. Srinivasan, K. G. Casey, B. Braren, and M. Yeh, J. Appl. Phys. 67, 1604 (1990).

${ }^{\text {s }}$ P. L. G. Venlzek, R. M. Gilgenbach, J. A. Sell, and D. M. Heffelfinger, J. Appl. Phys. 68, 965 (1990).

${ }^{9}$ A. Gupta, B. Braren, K. G. Casey, B. W. Hussey, and R. Kelly, App1. Phys. Lett. 59, 1302 (1991).
${ }^{10}$ J. Grun, J. Stamper, C. Manka, J. Resnick, R. Burris, J. Crawford, and B. H. Ripin, Phys. Rev. Lett. 66, 2738 (1991).

${ }^{11}$ J. Grun, J. Stamper, C. Manka, J. Resnick, R. Burris, and B. H. Ripin, Appl. Phys. Lett 59, 246 (1991).

${ }^{12}$ R. W. Dreyfus, R. Kelly, and R. E. Walkup, Appl. Phys. Lett. 49, 1478 (1986).

${ }^{13}$ H. Wang, A. P. Salzberg, and B. R. Weiner, Appl. Phys. Lett. 59, 935 (1991).

${ }^{14}$ S. Deshmukh, E. W. Rothe, and G. P. Reck, J. Appl. Phys. 66, 1370 (1989).

${ }^{15}$ M. A. Cappelli, P. H. Paul, and R. K. Hanson, Appl. Phys. Lett. 56, 1715 (1990).

${ }^{16}$ P. M. Goodwin and C. E. Otis, Appl. Phys. Lett. 55, 2286 (1989).

${ }^{17}$ R. E. Walkup, J. M. Jasinski, and R. W. Dreyfus, Appl. Phys. Lett. 48, 1691 (1986).

${ }^{18}$ D. B. Geohegan and D. N. Mashburn, Appl. Phys. Lett. 55, 2345 (1989).

${ }^{19}$ N. H. Cheung, Q. Y. Ying, J. P. Zheng, and H. S. Kwok, J. Appl. Phys. 69, 6349 (1991).

${ }^{20}$ H. Izumi, K. Ohata, T. Sawada, T. Morishita, and S. Tanaka, Appl. Phys. Lett. 59, 597 (1991).

${ }^{21}$ H. Izumi, K. Ohata, T. Sawada, T. Morishita, and S. Tanaka, Appl. Phys. Lett. 59, 2950 (1991).

${ }^{22}$ R. M. Gilgenbach and P. L. G. Ventzek, Appl. Phys. Lett. 58, 1597 (1991).

${ }^{23} \mathrm{~K}$. Horioka, N. Tazima, and K. Kazuya, Rev. Sci. Instrum. 61, 610 (1990).

${ }^{24}$ A. N. Mostovych, B. H. Ripin, and J. A. Stamper, Rev. Sci. Instrum. 59, 1497 (1988).

${ }^{25}$ R. Kelly, Nucl. Instrum. Methods B 46, 441 (1990).

${ }^{26}$ E. F. Gabl, B. H. Failor, C. J. Armentrout, N. D. Delameter, W. B. Fechner, R. A. Bosch, G. E. Busch, Z. M. Koenig, D. Ress, L. Suter, and R. J. Schroeder, Phys. Rev. Lett. 63, 2737 (1989).

${ }^{27}$ P. E. Dyer and J. Sidhu, J. Appl. Phys. 64, 4657 (1988).

${ }^{28}$ B. Ranby and J. F. Rabek, Photodegradation, Photo-oxidation and Photostabilization of Polymers: Principles and Applications (Wiley, New York, 1975).

${ }^{29}$ This was brought to our attention by the referee.

${ }^{30}$ Ya B. Zel'dovich and Yu P. Raizer, Physics of Shock Waves and High Temperature Hydrodynamic Phenomena (Academic, New York, 1966), p. 93.

${ }^{31}$ D. M. Mann and D. P. Weaver, Air Force Rocket Propulsion Laboratory, Edwards Air Force Base Report, AFRPL TR-84-053, August, 1984 (unpublished).

${ }^{32}$ R. E. Treybal, Mass-Transfer Operations, 3rd ed. (McGraw-Hill, New York, 1980).

${ }^{33}$ A. D. Sappey and T. K. Gamble, Appl. Phys. B 53, 353 (1991).

${ }^{34}$ I. Yamada, H. Usui, and T. Takagi, J. Phys. Chem. 91, 2463 (1987).

${ }^{35}$ M. Raleigh, NRL Memorandum Report 4555, August 24 (1981).

${ }^{36}$ S. Kuper and J. Bramnon, Appl. Phys. Lett. 60, 1633 (1992). 